\title{
Negligible Isotopic Effect on Dissociation of Hydrogen Bonds
}

Chuanqi Ge ${ }^{1,2} \boldsymbol{q}$, Y uneng Shen ${ }^{2,5} \boldsymbol{q}$, Gang-Hua Deng ${ }^{2}$, Yuhuan Tian ${ }^{2}$, Dongqi Yu ${ }^{1}$, Xueming Yang ${ }^{2}$, Kaijun Yuan ${ }^{2}$, Junrong Zheng ${ }^{2,3,4 *}$

${ }^{1}$ School of Physics and Electronic Technology, Liaoning Normal University, Dalian 116029, China

${ }^{2}$ State Key Laboratory of Molecular Reaction Dynamics, Dalian Institute of the Chemical Physics, ChineseAcademy of Sciences, Dalian 116023, Liaoning, China

${ }^{3}$ Department of Chemistry, Rice University, Houston, Texas, USA

${ }^{4}$ College of Chemistry and Molecular Engineering, Beijing National Laboratory for Molecular Sciences, Peking University, Beijing 100871, China

${ }^{5}$ University of the Chinese Academy of Sciences, Beijing 100049, China

\The authors have equal contribution.

*To whom correspondence should be addressed:

kjyuan@dicp.ac.cn; junrong@rice.edu 


\section{Supporting Information}

\section{Calculating Equilibrium Constant}

The followings are the chemical properties of solutes and solvents in our experiments

phenol:

MW: $94.1 \mathrm{~g} / \mathrm{mol}$;

p-xylene:

MW: $106.2 \mathrm{~g} / \mathrm{mol}$; density: $0.861 \mathrm{~g} / \mathrm{mL}$

mesitylene:

MW:120.2 g/mol; density: $0.8675 \mathrm{~g} / \mathrm{mL}$

$\mathrm{CCl}_{4}$ :

MW: $153.8 \mathrm{~g} / \mathrm{mol}$;density: $1.594 \mathrm{~g} / \mathrm{mL}$

1.1 The complex/free concentration ratio in phenol-OD/p-xylene/ $\mathrm{CCl}_{4}(0.6 \mathrm{wt} \%$, 1:7 wt) 

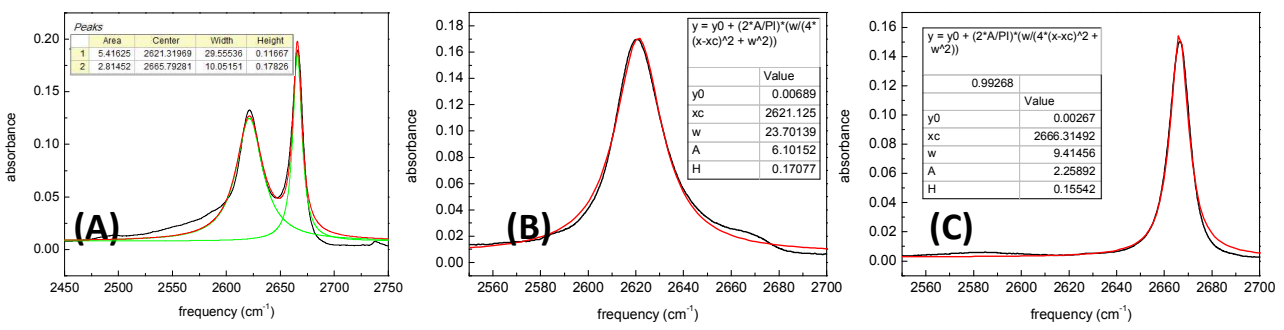

Figure S1.(A) FTIR absorption spectrum of the ODstretch of phenol-OD (hydroxyl H replaced with $D)$ in the mixed p-xylene/CCl solvent $(0.6 w t \%, 1: 7 w t)$, which displays absorptions for both free and complexed phenol-OD (black curve).Complexed OD was fitted by lorentzcurve displayed with red color. Free ODwas fitted by lorentz curve displayed with green color.(B) FTIR absorption spectrum of the OD stretch of phenol-OD in p-xylene (0.6 wt\%,phenol-p-xylene complex, black curve), and complexed $O D$ was fitted by lorentz curve displayed with red color. (C)FTIR absorption spectrum of the ODstretch of phenol-OD in $\mathrm{CCl}_{4}(0.2 w t \%$,free phenol-OD, black curve), and free OD was fitted by lorentz curve displayed with red color.

$$
\begin{aligned}
& \frac{I_{F T I R}^{m-2621}}{I_{F T I R}^{p-2621}}=\frac{k C_{1}^{2621} \mu_{2621}^{2}}{k C_{2}^{2621} \mu_{2621}^{2}} \\
& \frac{I_{F T I R}^{m-2666}}{I_{F T I R}^{p-2666}}=\frac{k C_{1}^{2666} \mu_{2666}^{2}}{k C_{2}^{2666} \mu_{2666}^{2}} \\
& C_{1}^{2621}: C_{1}^{2666}=\frac{I_{F T I R}^{m-2621}}{I_{F T I R}^{p-2621} / C_{2}^{2621}}: \frac{I_{F T I R}^{m-2666}}{I_{F T I R}^{p-666} / C_{2}^{2666}} \\
& C_{2}^{2621}=\frac{0.6 / 95.1}{100 / 0.861}=5.432 \times 10^{-5} \\
& C_{2}^{2666}=\frac{0.2 / 95.1}{100 / 1.594}=3.352 \times 10^{-5}
\end{aligned}
$$


$C_{1}^{2621}: C_{1}^{2666}=\frac{5.42}{6.1} \times 5.432 \times 10^{-5}: \frac{2.81}{2.26} \times 3.352 \times 10^{-5} \approx 1.17$

1.2 The complex/free concentration ratio in phenol/p-xylene/ $\mathrm{CCl}_{4}(0.6 \mathrm{wt} \%, 1: 7$ wt)
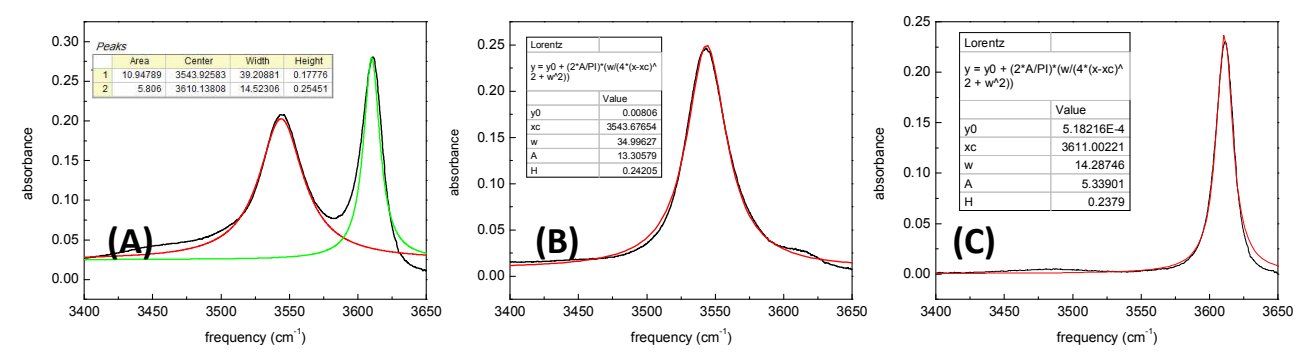

Figure S2.(A) FTIR absorption spectrum of the OHstretch of phenolin the mixed p-xylene/CCl $\mathrm{Cl}_{4}$ solvent $(0.6 \mathrm{wt} \%, 1: 7 \mathrm{wt})$, which displays absorptions for both free and complexed phenol (black curve).Complexed OH was fitted by lorentz curve displayed with red color. Free OHwas fitted by lorentz curve displayed with green color. (B) FTIR absorption spectrum of the OH stretch of phenol in p-xylene (0.6 wt\%,phenol-p-xylene complex, black curve), and complexed OH was fitted by lorentz curve displayed with red color. (C)FTIR absorption spectrum of the OHstretch of phenolin $\mathrm{CCl}_{4}(0.2 w t \%$, free phenol, black curve), and free $\mathrm{OH}$ was fitted by lorentz curve displayed with red color.

$$
\begin{aligned}
& C_{1}^{3543}: C_{1}^{3610}=\frac{I_{F T I R}^{m-3543}}{I_{F T I R}^{p-3543} / C_{2}^{3543}}: \frac{I_{F T I R}^{m-3610}}{I_{F T I R}^{p-3610} / C_{2}^{3610}} \\
& C_{2}^{3543}=\frac{0.6 / 94.1}{100 / 0.861}=5.49 \times 10^{-5} \\
& C_{2}^{3610}=\frac{0.2 / 94.1}{100 / 1.594}=3.388 \times 10^{-5}
\end{aligned}
$$




$$
C_{1}^{3543}: C_{1}^{3610}=\frac{10.95}{13.31} \times 5.49 \times 10^{-5}: \frac{5.81}{5.34} \times 3.388 \times 10^{-5} \approx 1.22
$$

\subsection{The complex/free concentration ratio in phenol-OD/mesitylene/ $\mathrm{CCl}_{4}(0.6$}

wt\%, 1:7 wt)
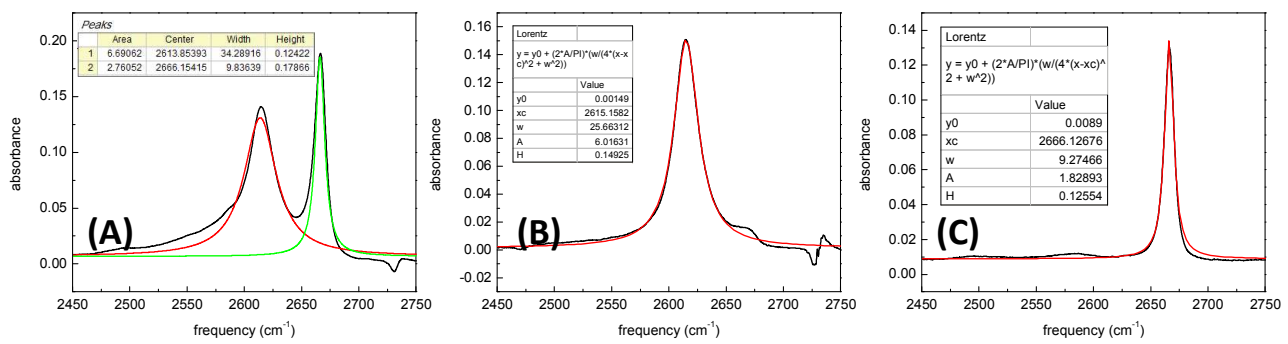

Figure S3.(A) FTIR absorption spectrum of the ODstretch of phenol-OD (hydroxyl H replaced with $D)$ in the mixed mesitylene/CCl 4 solvent $(0.6 w t \%, 1: 7 w t)$, which displays absorptions for both free and complexed phenol-OD (black curve).Complexed $O D$ was fitted by lorentz curve displayed with red color. Free ODwas fitted by lorentz curve displayed with green color. (B) FTIR absorption spectrum of the OD stretch of phenol-OD inmesitylene $(0.6 \mathrm{wt} \%$,phenol-p-xylene complex, black curve), and complexed OD was fitted by lorentz curve displayed with red color. (C)FTIR absorption spectrum of the ODstretch of phenol-OD in $\mathrm{CCl}_{4}$ (0.2wt\%, free phenol-OD, black curve), and free $O D$ was fitted by lorentz curve displayed with red color.

$$
\begin{aligned}
& \frac{I_{F T I R}^{m-2615}}{I_{F T I R}^{p-2615}}=\frac{k C_{1}^{2615} \mu_{2615}^{2}}{k C_{2}^{2615} \mu_{2615}^{2}} \\
& \frac{I_{F T I R}^{m-2666}}{I_{F T I R}^{p-2666}}=\frac{k C_{1}^{2666} \mu_{2666}^{2}}{k C_{2}^{2666} \mu_{2666}^{2}}
\end{aligned}
$$




$$
\begin{aligned}
& C_{1}^{2615}: C_{1}^{2666}=\frac{I_{F T I R}^{m-2615}}{I_{F T I R}^{p-2615} / C_{2}^{2615}}: \frac{I_{F T I R}^{m-2666}}{I_{F T I R}^{p-2666} / C_{2}^{2666}} \\
& C_{2}^{2615}=\frac{0.6 / 95.1}{100 / 0.8675}=5.473 \times 10^{-5} \\
& C_{2}^{2666}=\frac{0.2 / 95.1}{100 / 1.594}=3.352 \times 10^{-5} \\
& C_{1}^{2615}: C_{1}^{2666}=\frac{6.69}{6.02} \times 5.473 \times 10^{-5}: \frac{2.76}{1.83} \times 3.352 \times 10^{-5} \approx 1.2
\end{aligned}
$$

\subsection{The complex/free concentration ratio in phenol/mesitylene/ $\mathrm{CCl}_{4}(0.6 \mathrm{wt} \%$,}

\section{$1: 7 \mathrm{wt})$}
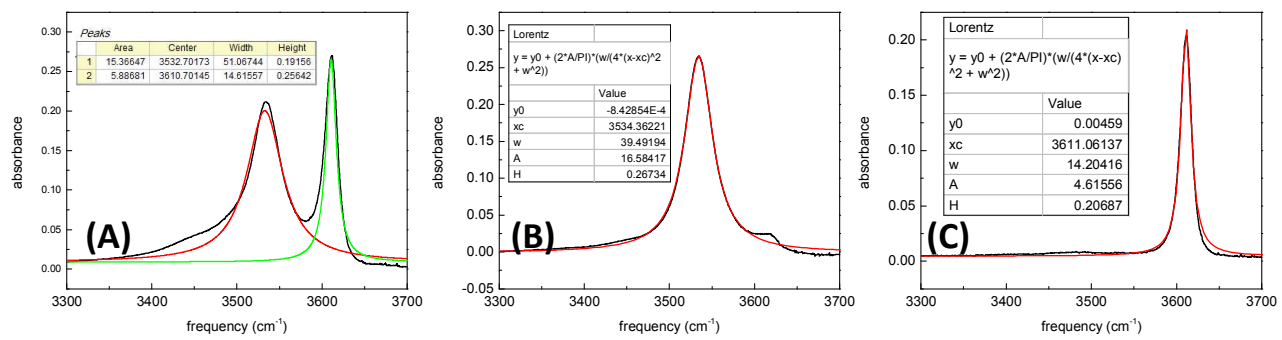

Figure S4.(A) FTIR absorption spectrum of the OHstretch of phenol in the mixed mesitylene/CCl $\mathrm{Cl}_{4}$ solvent $(0.6 \mathrm{wt} \%, 1: 7 \mathrm{wt})$, which displays absorptions for both free and complexed phenol (black curve).Complexed $\mathrm{OH}$ was fitted by lorentz curve displayed with red color. Free OHwas fitted by lorentz curve displayed with green color. (B) FTIR absorption spectrum of the OH stretch of phenol inmesitylene (0.6 wt\%,phenol-mesitylene complex, black curve), and complexed $\mathrm{OH}$ was fitted by lorentz curve displayed with red color. (C)FTIR absorption spectrum of the OHstretch of phenol in $\mathrm{CCl}_{4}(0.2 w t \%$, free phenol, black curve), and free $\mathrm{OH}$ was fitted by lorentz curve displayed with red color. 


$$
\begin{aligned}
& C_{1}^{3533}: C_{1}^{3611}=\frac{I_{F T I R}^{m-3533}}{I_{F T I R}^{p-3532} / C_{2}^{3533}}: \frac{I_{F T I R}^{m-3611}}{I_{F T I R}^{p-3611} / C_{2}^{3611}} \\
& C_{2}^{3533}=\frac{0.6 / 94.1}{100 / 0.8675}=5.531 \times 10^{-5} \\
& C_{2}^{3611}=\frac{0.2 / 94.1}{100 / 1.594}=3.388 \times 10^{-5} \\
& C_{1}^{3533}: C_{1}^{3611}=\frac{15.37}{16.58} \times 5.531 \times 10^{-5}: \frac{5.89}{4.62} \times 3.388 \times 10^{-5} \approx 1.19
\end{aligned}
$$

\section{The method for obtaining the time dependent populations from the chemical exchange measurements}

The method has been described in our previous study. ${ }^{1-5}$ The data were fitted in the following manner. For the peak intensities at $0 \mathrm{ps}$, the population ratio of the two species is taken to be the same as the complex/free concentration ratio obtained from the FTIR measurements. Because at 0 ps the chemical exchange has not occurred. All of the diagonal peaks for the complexes are divided by the intensity of the complex peak at $0 \mathrm{ps}$ to yield the time-dependent populations for complex species (CC). All of the free diagonal peaks are divided by the intensity of the free peak at 0 ps and then divided by the equilibrium constant to give the time-dependent populations of free species (FF). This procedure accounts for the fact that the transition dipole moments of the complex and free species are not the same. The diagonal peak amplitudes depend on the fourth power of their respective transition dipoles. The off-diagonal peak intensities depend on the product of the squares of the transition dipoles of the complex and free species. To take this into account, the cross-peaks are divided by the square root of the intensity ratio of the complexed peak 
intensity over the free peak intensity (complexed and free peak intensity are normalized by the pump power respectively) divided by the complex/free concentration ratio.

\section{The free diagonal peak dynamics in pure solvent}

In main text, the free diagonal peak data in mixed solvent $(0.6 \mathrm{wt} \%$ phenol in the mixed p-xylene/ $\mathrm{CCl}_{4}$ solvent $\left.(1: 7 \mathrm{wt})\right)$ has the recurrence on its experimental cure at 12 ps. Meanwhile, its blue peak also has the recurrence (see figure S5). To determine if it will affect the dissociation time, we choose to measure the free diagonal peak data in pure solvent $\left(0.6 \mathrm{wt} \%\right.$ phenol in $\mathrm{CCl}_{4}$ solvent). As can be seen in figure $\mathrm{S} 6 \& 7$, we find that not only the free diagonal peak data in mixed solvent has the recurrence on its experimental cure at $12 \mathrm{ps,} \mathrm{but} \mathrm{also} \mathrm{the} \mathrm{free} \mathrm{diagonal} \mathrm{peak} \mathrm{data} \mathrm{in} \mathrm{pure} \mathrm{solvent} \mathrm{has}$ the same phenomenon. Moreover, from Zheng's previous studies, the dissociation time is mainly determined by the growth of the off-diagonal peaks, and the missed fit

for the free diagonal peak affect it little. ${ }^{3}$ Therefore this phenomenon will not affect the dissociation time. 


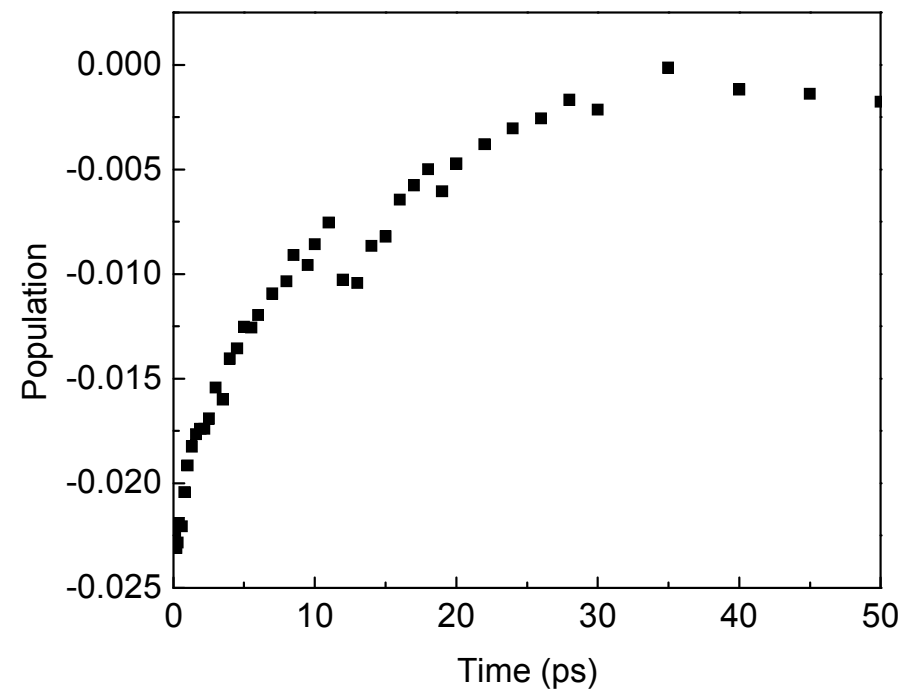

Figure S5. Rotation-free population decay of phenol in p-xylene/CCl $\mathrm{Cl}_{4}$ mixture at 3441 $\mathrm{cm}^{-1}$ (blue peak).

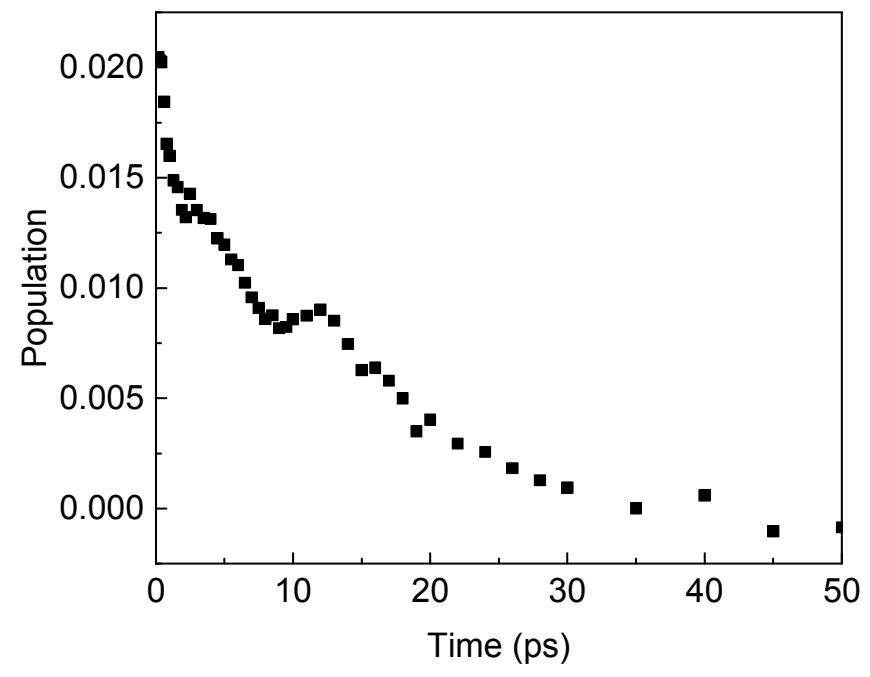

Figure S6. Rotation-free population decay of phenol in $\mathrm{CCl}_{4}$ at $3604 \mathrm{~cm}^{-1}$ (red peak). 


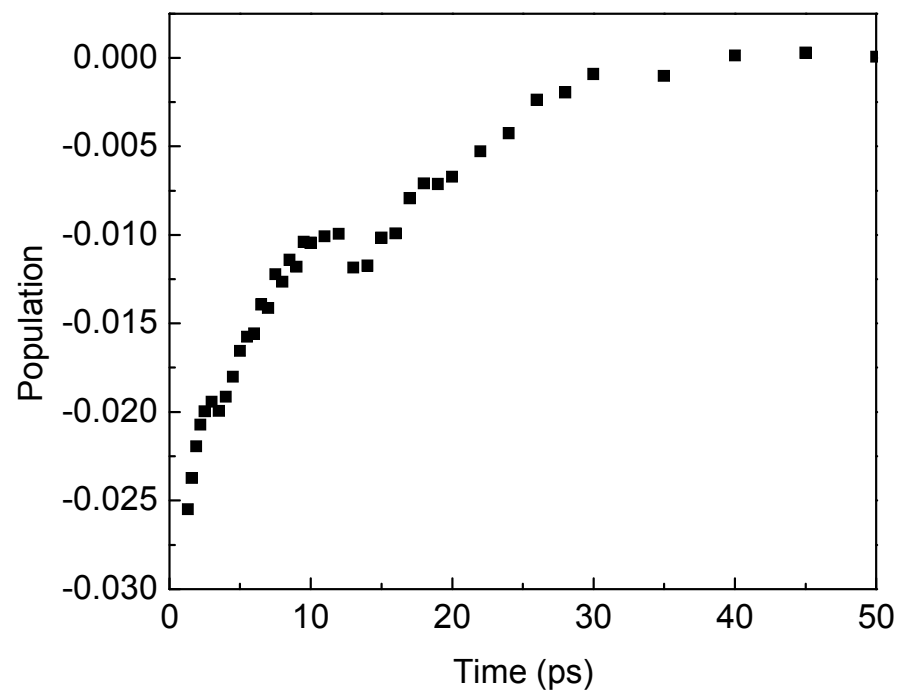

Figure S7. Rotation-free population decay of phenol in $\mathrm{CCl}_{4}$ at $3441 \mathrm{~cm}^{-1}$ (blue peak).

\section{The peak intensity data for the 0-1 transition region for the four mixtures}
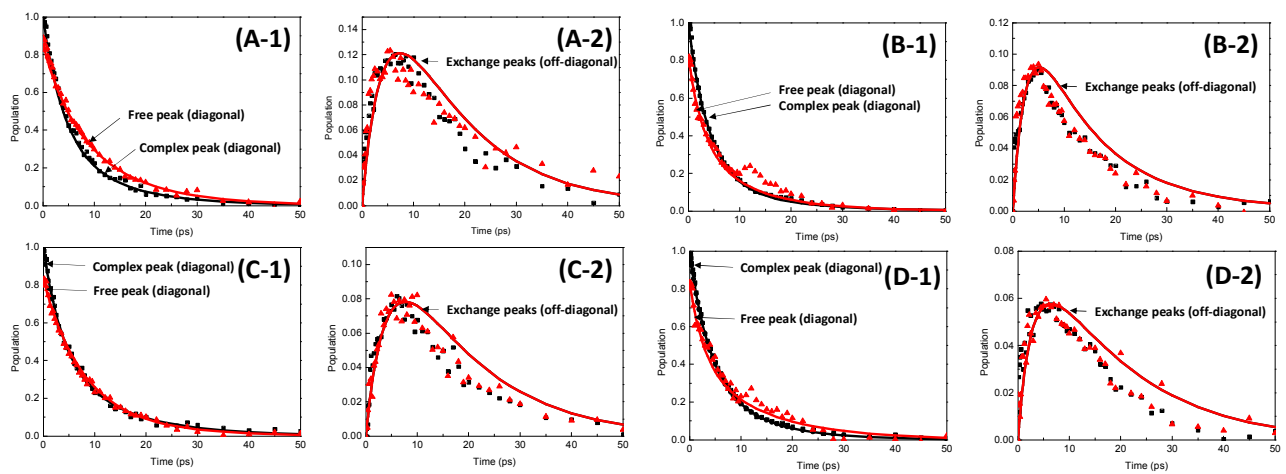

Figure S6. Time dependent data (symbols) of the two diagonal ( $X-1, X=A, B, C$ or D) and two off-diagonal $(X-2)$ peak intensities in the 0-1 portion of the pump probe vibrational spectra for (A) phenol-OD in the p-xylene/CCl $C_{4}$ mixture, (B) phenol in the p-xylene/CCl $\mathrm{Cl}_{4}$ mixture, $(\mathrm{C})$ phenol-OD in the mesitylene/CCl 4 mixture and $(D)$ phenol in the mesitylene/CCl $\mathrm{Cl}_{4}$ mixture. In each figure, we use the solid curves calculated from the chemical exchange kinetic model with one adjustable parameter, $k_{d}$, to fit the 
experimental data. Other parameters in the model are determined experimentally.

Using these parameters, we can obtain

In the main text, the drawing format followed Junrong's previous study and it is easy for us to compare with the previous work. However, in this place, we scale our cross-peak data to make the readers more earnestly assess the accuracy of our model.

\section{Pictures for vibrations for the two systems}

For phenol/p-xylene complex and phenol-OD/p-xylene complexes, DFT calculations show that these two complexes have same structure, same hydrogen bond mode and identical binding energy. In table 2 (see main text) we list all of the possible vibrations that can be involved in hydrogen bond dissociation for them. And in figure S7, we list the corresponding pictures for vibrations for phenol-OD/p-xylene complexes. 

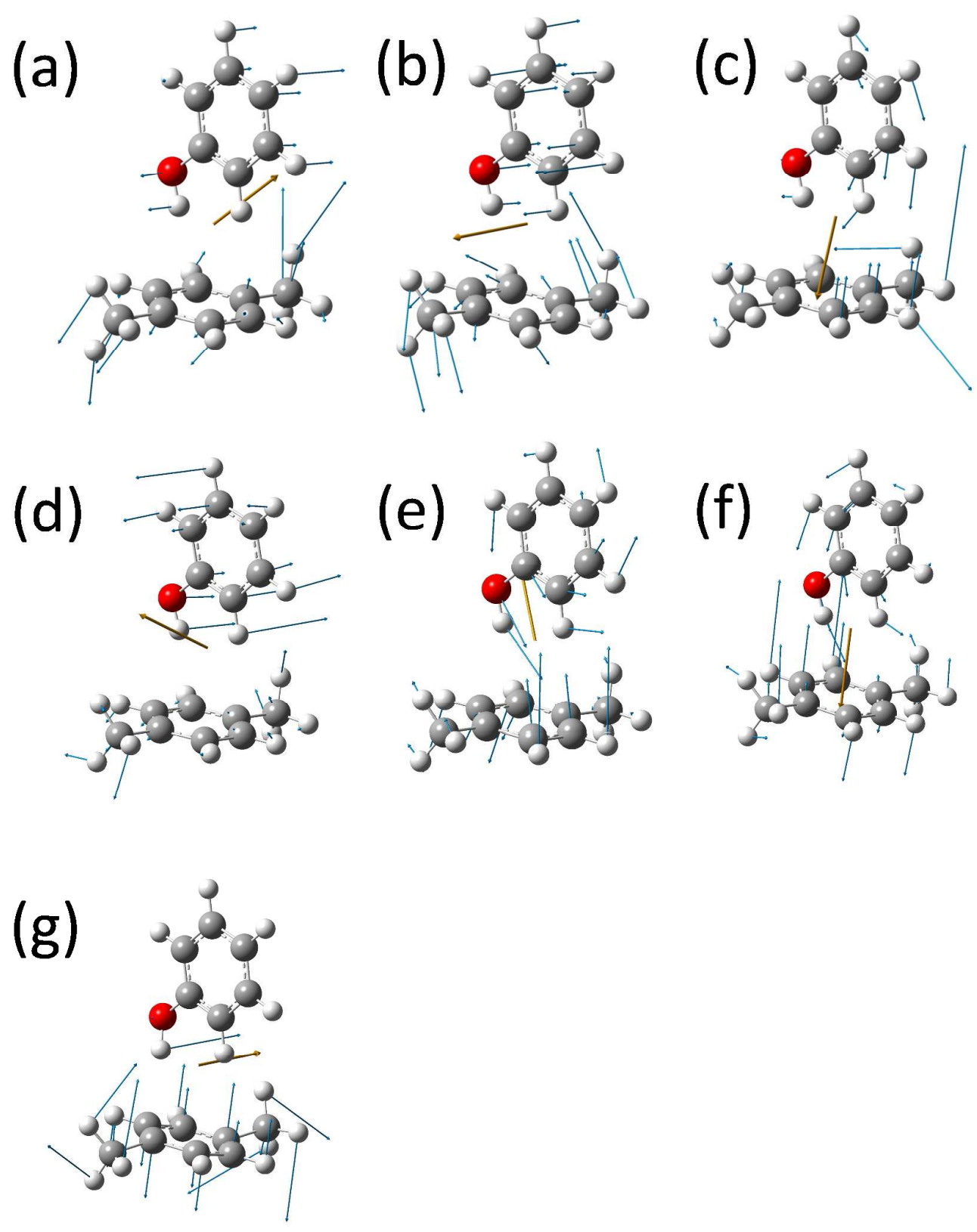

Figure S7. The corresponding pictures for vibrations for phenol-OD/p-xylene complexes.(a) $12.28 \mathrm{~cm}^{-1}$. (b) $15.89 \mathrm{~cm}^{-1}$. (c) $30.42 \mathrm{~cm}^{-1}$. (d) $37.79 \mathrm{~cm}^{-1}$. (e) $43.32 \mathrm{~cm}^{-1}$. (f) $71.2 \mathrm{~cm}^{-1}$. (g) $308.59 \mathrm{~cm}^{-1}$. The blue arrows show displacement vectors of atoms, and the yellow arrow show dipole derivative unit vector.

For phenol/mesitylene complex and phenol-OD/mesitylene complexes, DFT calculations show that these two complexes have same structure, same hydrogen bond 
mode and identical binding energy. In table 3 (see main text) we list all of the possible vibrations that can be involved in hydrogen bond dissociation for them. And in figure S8, we list the corresponding pictures for vibrations for phenol-OD/mesitylene complexes.
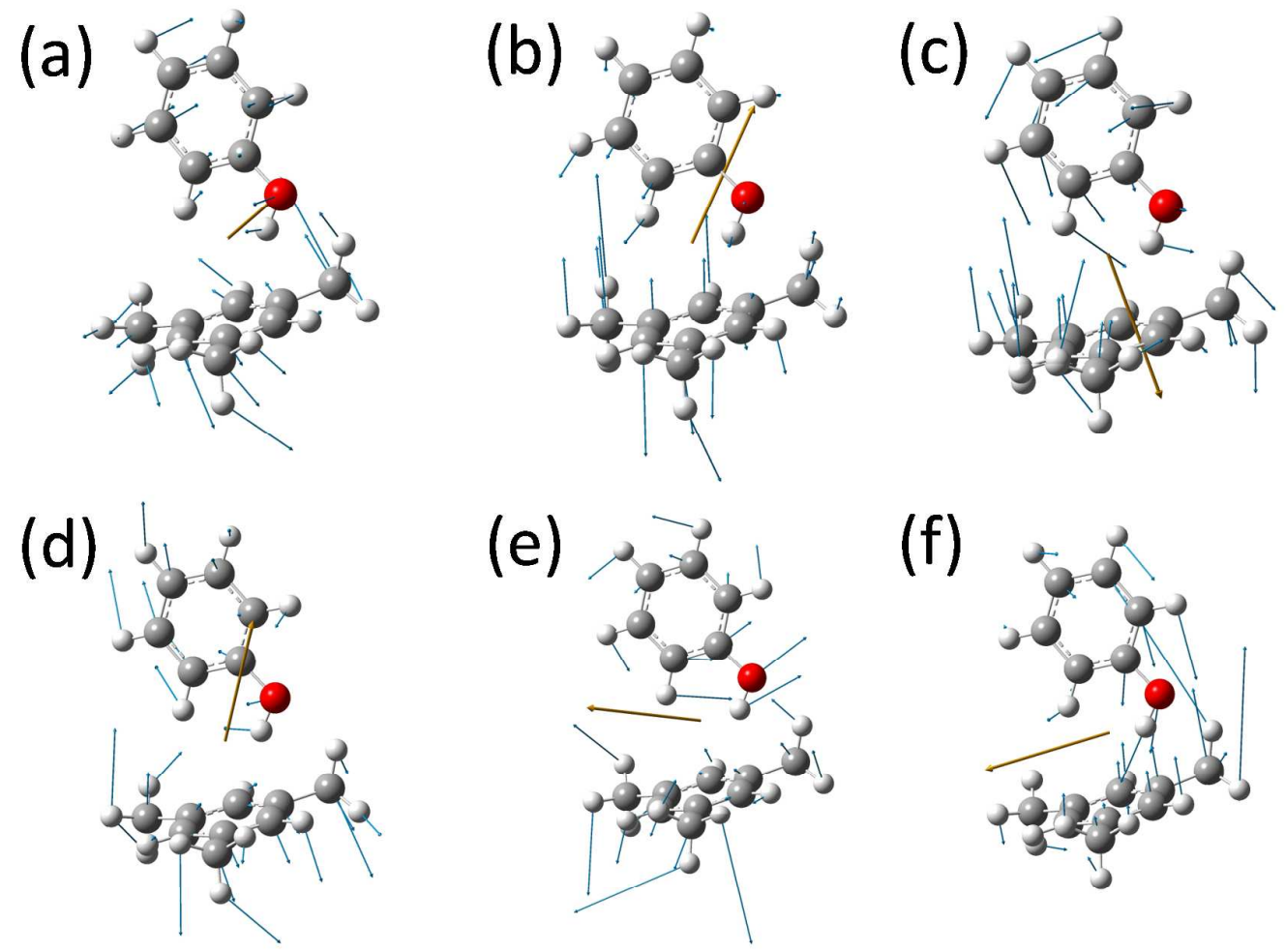

(f)
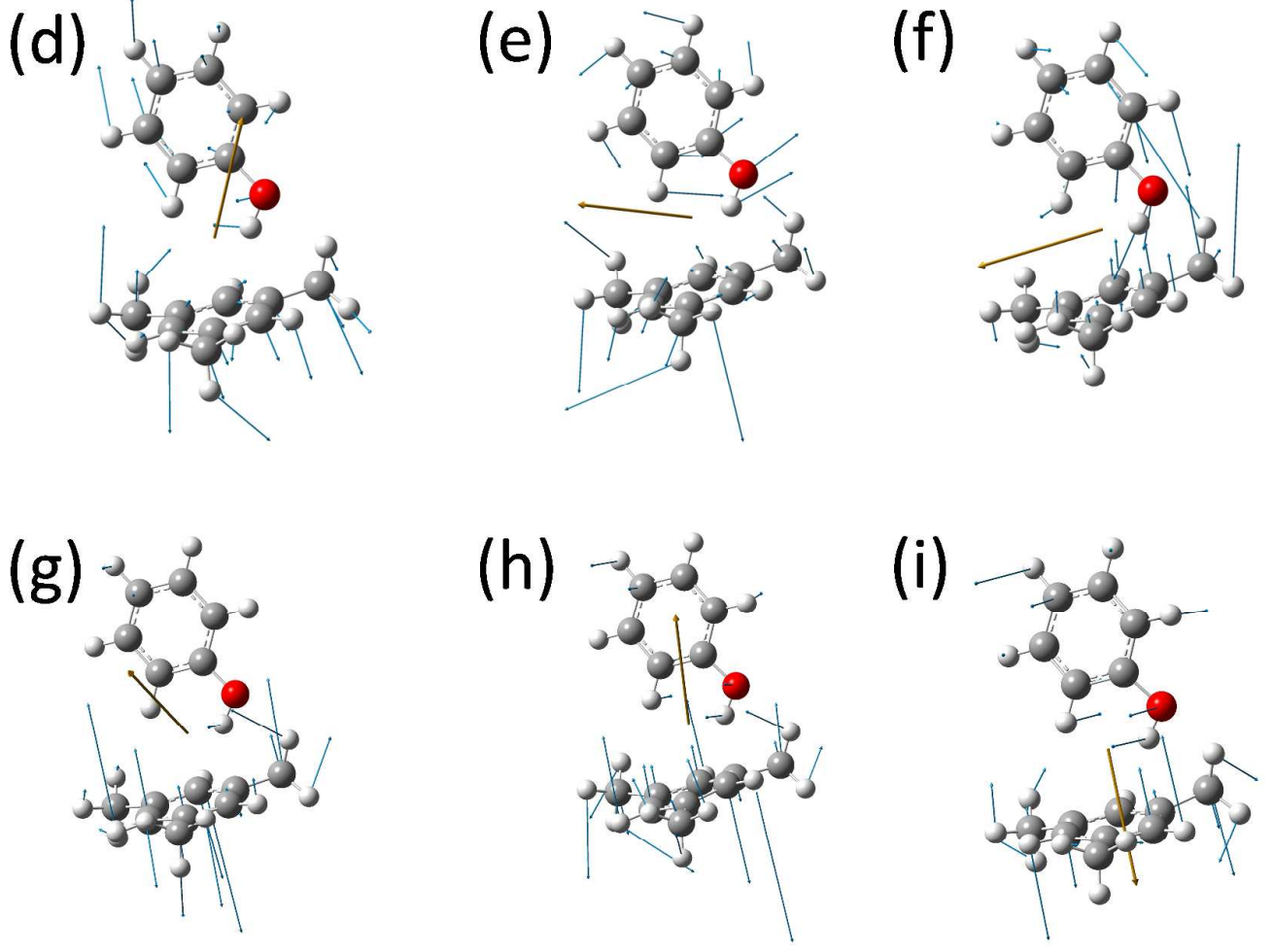

Figure S8. The corresponding pictures for vibrations for phenol-OD/mesitylene complexes.(a) $13.49 \mathrm{~cm}^{-1}$. (b) $21.28 \mathrm{~cm}^{-1}$. (c) $28.48 \mathrm{~cm}^{-1}$. (d) $37.35 \mathrm{~cm}^{-1}$. (e) $52.02 \mathrm{~cm}^{-1}$. (f) $81.87 \mathrm{~cm}^{-1}$. (g) $225.22 \mathrm{~cm}^{-1}$. (h) $232.99 \mathrm{~cm}^{-1}$. (i) $235.78 \mathrm{~cm}^{-1}$. The blue arrows show 
displacement vectors of atoms, and the yellow arrow show dipole derivative unit vector.

\section{Calculations in different solvents with different basis sets}

The DFT calculations in main text were mainly to show that $\mathrm{H} / \mathrm{D}$ exchange does not cause significant changes in the frequencies of those low frequency vibrations for a phenol/benzene derivative complex (so that the issue of zero point energy can be ignored). Whether the calculated structures of complexes in gas phase are similar to those in real liquids is in some sense irrelevant. However, this issue is worth further discussion. Intuitively, the calculated structures in this way should be quite different from those liquids. However, MD simulations show that they are actually quite similar. ${ }^{2,6}$ Gaussian calculations can produce relative large uncertainties for those low vibrational frequencies. However, our purpose is to compare the difference between $\mathrm{H}$ and $\mathrm{D}$, not their individual absolute values. We expect that the uncertainties will be offset because the uncertainties are expected to be similar for both $\mathrm{H}$ and $\mathrm{D}$. Calculations in different solvents with different basis sets (table S1-S4) also show similar results for the comparison.

Table S1.Vibrations of phenol-p-xylene and phenol-OD-p-xylene complexes that can be involved in hydrogen bond dissociation. The phenol-p-xylene complex or phenol-OD-p-xylene complex calculated with DFT at PCM/B3LYP/6-31+G(d,p) level for molecules in CarbonTetraChloride solution.

\begin{tabular}{|l|l|l|l|l|l|l|l}
\hline Vibrations $\left(\mathrm{cm}^{-1}\right)$ & 1 & 2 & 3 & 4 & 5 & 6 & 7 \\
\hline
\end{tabular}




\begin{tabular}{cccccccc}
\hline Phenol/p-xylene & 12.84 & 18.88 & 29.73 & 39.63 & 47.24 & 72.54 & 310.34 \\
phenol-OD/p-xylene & 12.94 & 18.74 & 30.13 & 38.34 & 46.49 & 72.33 & 308.45 \\
\hline
\end{tabular}

Table S2.Vibrations of phenol-p-xylene and phenol-OD-p-xylene complexes that can be involved in hydrogen bond dissociation. The phenol-p-xylene complex or phenol-OD-p-xylene complex calculated with DFT at PCM/B3LYP/6-31+G(d,p) level for molecules in p-Xylene solution.

\begin{tabular}{cll|l|l|l|l|l|l}
\hline Vibrations $\left(\mathrm{cm}^{-1}\right)$ & 1 & 2 & 3 & 4 & 5 & 6 & 7 \\
\hline Phenol/p-xylene & 12.82 & 18.91 & 29.68 & 39.65 & 47.31 & 72.55 & 310.34 \\
phenol-OD/p-xylene & 12.78 & 18.85 & 29.61 & 39.31 & 46.91 & 72.21 & 308.54 \\
\hline
\end{tabular}

Table S3.Vibrations of phenol-p-xylene and phenol-OD-p-xylene complexes that can be involved in hydrogen bond dissociation. The phenol-p-xylene complex or phenol-OD-p-xylene complex calculated with DFT at PCM/B3LYP/6-311++G(d,p) level for molecules in CarbonTetraChloride solution.

\begin{tabular}{clll|l|l|l|l|l}
\hline Vibrations $\left(\mathrm{cm}^{-1}\right)$ & 1 & 2 & 3 & 4 & 5 & 6 & 7 \\
\hline Phenol/p-xylene & 13.21 & 18.95 & 29.64 & 36.97 & 48.38 & 71.04 & 309.00 \\
phenol-OD/p-xylene & 13.23 & 19.11 & 29.61 & 36.71 & 48.06 & 70.08 & 306.21 \\
\hline
\end{tabular}

Table S4.Vibrations of phenol-p-xylene and phenol-OD-p-xylene complexes that can be involved in hydrogen bond dissociation. The phenol-p-xylene complex or phenol-OD-p-xylene complex calculated with DFT at PCM/B3LYP/6-311++G(d,p) 
level for molecules in p-Xylene solution.

\begin{tabular}{cll|l|l|l|l|l|l}
\hline Vibrations $\left(\mathrm{cm}^{-1}\right)$ & 1 & 2 & 3 & 4 & 5 & 6 & 7 \\
\hline Phenol/p-xylene & 13.24 & 19.21 & 29.67 & 37.02 & 48.54 & 71.15 & 309.02 \\
phenol-OD/p-xylene & 13.20 & 19.14 & 29.57 & 36.71 & 48.13 & 70.81 & 306.21 \\
\hline
\end{tabular}

\section{References}


(1) Zheng, J. R.; Kwak, K.; Chen, X.; Asbury, J. B.; Fayer, M. D. Formation and Dissociation of Intra-Intermolecular Hydrogen-Bonded Solute-Solvent Complexes: Chemical Exchange Two-Dimensional Infrared Vibrational Echo Spectroscopy. J. Am. Chem. Soc. 2006, 128, 2977-2987.

(2) Zheng, J.; Fayer, M. D. Hydrogen Bond Lifetimes and Energetics for Solute/Solvent Complexes Studied with 2d-Ir Vibrational Echo Spectroscopy. J. Am. Chem. Soc. 2007, 129, 4328-4335.

(3) Zheng, J.; Fayer, M. D. Solute-Solvent Complex Kinetics and Thermodynamics Probed by 2d-Ir Vibrational Echo Chemical Exchange Spectroscopy. J. Phys. Chem. B 2008, 112, $10221-10227$.

(4) Bian, H.; Li, J.; Wen, X.; Zheng, J. Mode-Specific Intermolecular Vibrational Energy Transfer. I. Phenyl Selenocyanate and Deuterated Chloroform Mixture. $J$. Chem. Phys. 2010, 132, 184505.

(5) Bian, H.; Wen, X.; Li, J.; Zheng, J. Mode-Specific Intermolecular Vibrational Energy Transfer. Ii. Deuterated Water and Potassium Selenocyanate Mixture. J. Chem. Phys. 2010, 133, 034505.

(6) Kwac, K.; Lee, C.; Jung, Y.; Han, J.; Kwak, K.; Zheng, J.; Fayer, M. D.; Cho, M. Phenol-Benzene Complexation Dynamics: Quantum Chemistry Calculation, Molecular Dynamics Simulations, and Two Dimensional Ir Spectroscopy. J. Chem. Phys. 2006, 125, 244508. 